\title{
Erratum to: Introduction to Partial Differential Equations
}

\section{Erratum to:}

D. Borthwick, Introduction to Partial Differential Equations, Universitext, http://doi.org/10.1007/978-3-319-48936-0

In the original version of the book, the belated corrections from author for Chaps. 2, 3, 4, 6 and 11 have been incorporated.

The updated online version of these chapters can be found at http://doi.org/10.1007/978-3-319-48936-0

http://doi.org/10.1007/978-3-319-48936-0_2

http://doi.org/10.1007/978-3-319-48936-0_3

http://doi.org/10.1007/978-3-319-48936-0_4

http://doi.org/10.1007/978-3-319-48936-0_6

http://doi.org/10.1007/978-3-319-48936-0_11

(C) Springer International Publishing AG 2018

E1

D. Borthwick, Introduction to Partial Differential Equations,

Universitext, http://doi.org/10.1007/978-3-319-48936-0_14 